\title{
MORFOLOGIA DOS MÚSCULOS DA COXA DE MÃO-PELADA (Procyon cancrivorus) - CUVIER 1798
}

\author{
Firmino Cardoso Pereira, ${ }^{1}$ Vanessa Morais Lima ${ }^{2}$ e Kleber Fernando Pereira ${ }^{3}$ \\ 1. Graduando em Ciências Biológicas na Universidade Federal de Goiás. \\ E-mail: firminocpereira@gmail.com \\ 2. Pós-graduando em Anatomia Animal, Universidade de São Paulo \\ 3. Docente do Curso de Ciências Biológicas da Universidade Federal de Goiás.
}

\section{RESUMO}

O Procyon cancrivorus é um animal silvestre pertencente à ordem Carnivora, presente em todo o território brasileiro, alguns países da América Central, Uruguai e Argentina. Apesar de este animal estar fora da lista de animais em extinção, existem poucos dados morfológicos desta espécie. Na literatura brasileira e mundial, foram encontrados apenas estudos sobre seus hábitos, hábitat e alguns dados morfológicos. Propõe-se, neste trabalho, descrever as inserções proximais e distais dos músculos da coxa do $P$. cancrivorus, comparando-os com dados da literatura de carnívoros domésticos, como cães e gatos. Os cinco espécimes foram recolhidos pós-morte, vítimas de atropelamentos em rodovias. Procedeu-se à fixação dos animais em formaldeído (10\%) e ao armazenamento em cubas opacas tampadas. A descrição da musculatura da coxa foi possível por meio da técnica de dissecação, visualizando-se suas inserções proximais e distais, irrigação e inervação. Os resultados obtidos foram documentados com câmera fotográfica digital e comparados com dados da literatura dos músculos da coxa de animais domésticos, como cão e gato. Estudaram-se os músculos quadríceps femoral, sartório, tensor da fascialata, bíceps femoral, semitendinoso, adutores, semimembranoso, abdutor crural caudal, gêmeos, quadrado femoral, pectíneo e grácil, em que se constatou grande similaridade com os músculos da coxa dos carnívoros domésticos, como o cão e gato.

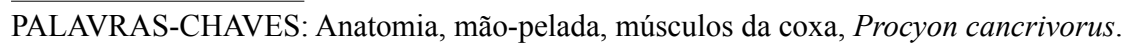

ABSTRACT

\section{MORPHOLOGY OF THE MUSCLES OF THE THIGH OF CRAB-EATING RACCOON (Procyon cancrivorus) - Cuvier 1798}

The Procyon cancrivorus is a wild animal, which belongs to the carnivore order, present in all Brazilian territory, some Central American countries, Uruguay and Argentina. Although this animal is not in the list of endangered species, there are few morphological data about this species. Only studies about the habits, habitat and some morphological data were found in Brazilian and world literature. The purpose of this study was to describe the proximal and distal insertions of the posterior and medial thigh muscles of the $P$. cancrivorus, comparing them with domestic carnivores literature data, such as dogs and cats. The five specimens were collected postmortem, victims of accidents on highways. The animals were fixed in formaldehyde (10\%) and stored in opaque covered tanks. The description of the thigh musculature was made possible by the dissection technique, visualizing its proximal and distal insertions, irrigation and innervation. The results were documented with a digital camera and compared with literature data of the thigh muscles of domestic animals such as cats and dogs. Studies on the quadriceps femoris, sartorius, tensor fascia lata, biceps femoris, semitendinosus, adductor, semimembranosus, caudal crural abductor, twins, quadratus femoris, gracilis and pectineus were carried out and great similarity with the thigh muscles of carnivores such as cats and dogs were found.

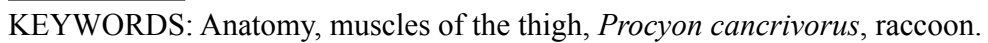




\section{INTRODUÇÃO}

O Procyon cancrivorus é agrupado ao filo Chordata, classe Mammalia, ordem Carnivora e família Procyonidae. Segundo RODRIGUES \& AURICCHIO (1994), no Brasil são encontradas quatro espécies de procionídeos: Nasua nasua (quati), Procyon cancrivorus, Potos flavus (jupara) e Bassaricyon gabbii (olingo). O quati e o P. cancrivorus possuem maior distribuição e são as mais conhecidas.

De ocorrência neotropical, $P$. cancrivorus é encontrado principalmente na Costa Rica, Bolívia, Colômbia, Guiana, Peru, Suriname, Venezuela, Trinindad e Tobago, Panamá, Uruguai, nordeste da Argentina (DÍAZ \& BARQUES, 2002; BARQUES et al., 2006) e em todos os biomas do território brasileiro, desde o Cerrado ao Pantanal, Mata Atlântica aos Campos Sulinos e Amazônia à Caatinga (CÂMARA \& MURTA, 2003; CUBAS et al., 2006). Existem registros de observação deste carnívoro silvestre também em mangues na região da Bahia, BA (FREITAS, 2005).

Muitos são os seus nomes populares, como guaxinim, rato lavador, urso lavador, mascarado, mão-lisa e principalmente mão-pelada, que se refere às mãos desprovidas de pelos, que deixam pegadas semelhantes às mãos de uma criança. Possui o tato bem desenvolvido e agilidade manual, permitindo-lhe procurar por peixes e outros organismos aquáticos em água rasa ou lodo, geralmente lavando-os antes de ingeri-los (CÂMARA \& MURTA, 2003; MURTA \& CÂMARA, 2006).

Segundo FREITAS (2005), o mão-pelada, maior procionídeo do Brasil, possui um comprimento corpóreo bastante variável - entre 40,0 e $100,0 \mathrm{~cm}$ - e a cauda longa apresentando anéis alternados, com 20,0 a $38,0 \mathrm{~cm}$. A coloração da pelagem varia do escuro ao grisalho, sendo prontamente identificado, dada a máscara preta que se estende dos olhos à base da mandíbula e em virtude da maior altura dos membros pélvicos, assim como a cauda peculiar (CÂMARA \& MURTA, 2003; CUBAS et al., 2006; MAMEDE, 2006; REIS et al., 2006). É considerado um potencial dispersor de sementes (WIESBAUER et al., 2008). Como observado por CAVALCANTI et al. (2009) no sertão de Pernambuco, o mão-pelada é um dos principais animais silvestres dispersores de sementes de imbuzeiro (Spondias tuberosa) naquela região.
Apesar de esta espécie estar fora da lista de animais em extinção (MACHADO et al., 2005; CUBAS et al., 2006), está entre as espécies de carnívoros silvestres brasileiros menos estudados. Poucos são os dados morfológicos desta espécie (MORATO et al., 2004). Constam-se, na literatura brasileira e internacional, apenas conhecimentos sobre hábito, hábitat e estudos de parâmetros sanguíneos (hematologia, bioquímica sanguínea, citoquímica sanguínea), biométricos, eletrocardiograma e doenças infecciosas e não infecciosas a que está sujeita a espécie (CUBAS et al., 2006). Há um estudo da topografia dos colaterais calibrosos do arco aórtico de um mão-pelada (SANTOS et al, 2004), outro estudo de ocorrência de criptorquidismo em P. canvrivorus de vida livre (DIAS et al., 2007), um estudo morfológico da glândula pineal (MARQUES et al., 2010) e um de microdesgaste dentário em $P$. cancrivorus (KOENEMANN, 2009). No que concerne às descrições morfológicas deste carnívoro, o presente grupo de pesquisa avaliou a anatomia dos músculos do braço (LIMA et al., 2009a), do antebraço (LIMA et al., 2010), da coxa (PEREIRA et al., 2010) e a distribuição da artéria mesentérica (LIMA et al., 2009b). E ainda, segundo JORGE et al. (2009), o mão-pelada está sujeito à inoculação pelo vírus da raiva, assim como outros animais selvagens.

Os músculos estabelecem o contorno morfológico característico de cada espécie e são os órgãos ativos do movimento (DI DIO et al., 2003). É dificil descrever o sistema muscular dos mamíferos, por causa das variações das disposições muscular nas diversas espécies (ORR, 1986). Mas é uma tarefa essencial, porque a caracterização anatômica dos músculos fornece informações relevantes sobre hábitos alimentares, força e comportamento (AVERSI-FERREIRA et al., 2006), diante dos escassos dados existentes na literatura especializada.

Os espécimes foram eventualmente coletados em rodovias, vítimas de atropelamentos, o que corrobora a citação de REIS et al. (2006), quando se referem que esta é a principal causa de morte da espécie. De acordo com LIMA et al. (2009a), há uma lacuna no que diz respeito à anatomia do Procyon cancrivorus, entre os vários carnívoros silvestres existentes, podendo ser comparada com a dos carnívoros domésticos, como o cão e o gato. Segundo HILDEBRAND \& GOSLOW (2006), as homologias quase sempre são evidentes 
entre os músculos de ordens e táxons inferiores, porém as descrições sobre a morfologia muscular desta espécie silvestre podem contribuir grandemente para o estabelecimento de um padrão normal, próprio ou não da espécie. Além disso, este estudo pode subsidiar futuras pesquisas nas diversas áreas correlatas. Assim, diante do escasso número de trabalhos publicados na literatura brasileira $\mathrm{e}$ internacional acerca da espécie, com o presente estudo morfológico objetivou-se estudar macroscopicamente os músculos do membro coxa de $P$. cancrivorus, no tocante às inserções distais e proximais, comparando-os com dados da literatura disponível acerca dessas estruturas em carnívoros domésticos, como cães e gatos.

\section{MATERIAL E MÉTODOS}

No desenvolvimento do trabalho foram utilizados cinco animais procedentes de coleta pós-morte em rodovias, vítimas de atropelamentos, cujos critérios obedeceram ao Comitê de Ética Institucional e à Lei Vigente (Lei 1.153/95), no ano de 2009. Os animais foram conduzidos ao Laboratório de Anatomia Humana e Comparativa da Universidade Federal de Goiás, Campus Jataí, onde se procedeu à canulação da aorta, perfusão com água aquecida $\left(40^{\circ} \mathrm{C}\right)$ e injeção de látex corado (neoprene 450, Dupont do Brasil e Sulvinil Corante, Glassuret S.A.), seguindo-se de fixação em solução de formaldeído (10\%). Posteriormente o material foi armazenado em cubas opacas tampadas, para evitar a penetração da luz e a evaporação do formol.

Para HILDEBRAND \& GOSLOW (2006), a dissecação é a técnica mais importante para o estudo dos músculos. Conhecer a posição anatômica de um músculo e suas relações com outros músculos e com o sistema esquelético é fundamental para interpretação. A descrição da musculatura dos espécimes possibilitou a visualização das inserções proximais e distais, irrigação e inervação do respectivo grupo muscular.

Posteriormente, os resultados obtidos foram documentados com câmera fotográfica digital (Sony Cybershot, 8.1 megapixels) e comparados com dados da literatura, acerca dos músculos do membro pélvico de animais domésticos, como cão e gato. Adotaram-se as respectivas designações anatômicas daqueles animais domésticos para as unidades do grupo muscular em estudo, obedecendo-se à INTERNATIONAL COMITEE ON VETERINARY GROSS ANATOMICAL NOMENCLATURE (2005).

\section{RESULTADOS}

Mediante consulta em literatura especializada, no tocante à musculatura da coxa de carnívoros domésticos, e observação dos músculos da coxa de $P$. cancrivorus, constataram-se as homologias musculares entre estas espécies. Os músculos da coxa deste carnívoro silvestre foram nomeados segundo as designações anatômicas.

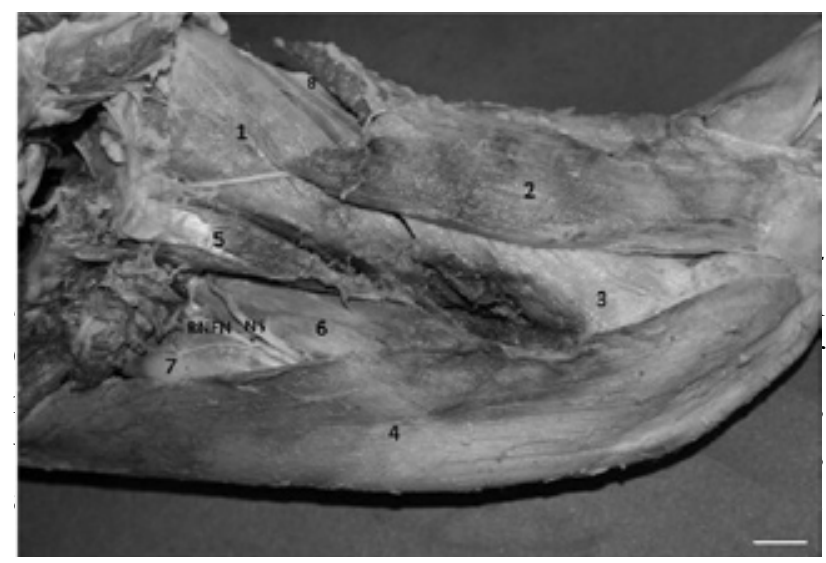

FIGURA 1. Vista medial da coxa direita de P. cancrivorus, onde estão situados os músculos: 1) adutor magno; 2) grácil; 3) semimembranoso; 4) sartório; 5) pectíneo; 6) vastomedial; 7) retofemoral; 8) semitendinoso, e NS) nervo safeno; RNF) ramo do nervo femoral. Barra $=0,5 \mathrm{~cm}$.

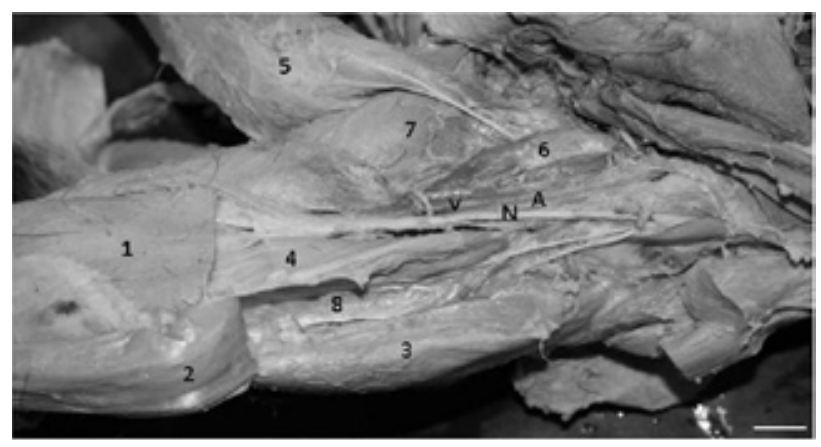

FIGURA 2. Vista medial da coxa esquerda de $P$. cancrivorus, estão situados os músculos: 1) sartório seccionado; 2) retofemoral seccionado; 3) vastolateral; 4) vastomedial; 5) grácil; 6) pectíneo; 7) adutor; 8) vasto intermédio; e AVN) artéria, veia e nervo safeno. Barra $=0,5 \mathrm{~cm}$.

Em vista lateral, identificaram-se os músculos: sartório (porção cranial), bíceps femoral, semitendinoso (porção cranial), tensor da fascialata, retofemo- 
ral, vastolateral, quadrado femoral e semimembranoso (porção cranial), evidenciados nas Figuras 3 e 4, respectivamente.

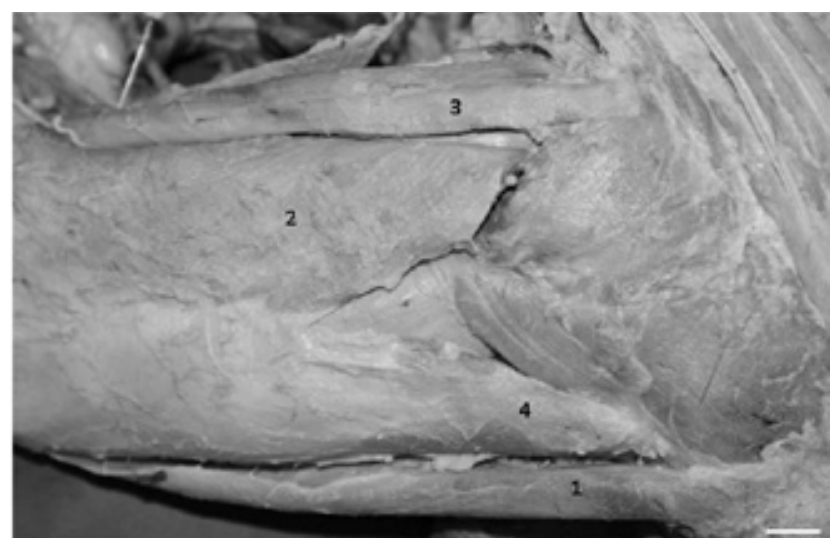

FIGURA 3. Vista lateral da coxa de $P$. cancrivorus, onde estão situados os músculos: 1) sartório; 2) bíceps femoral; 3) semitendinoso; 4) tensor da fascialata. Barra $=0,5 \mathrm{~cm}$.

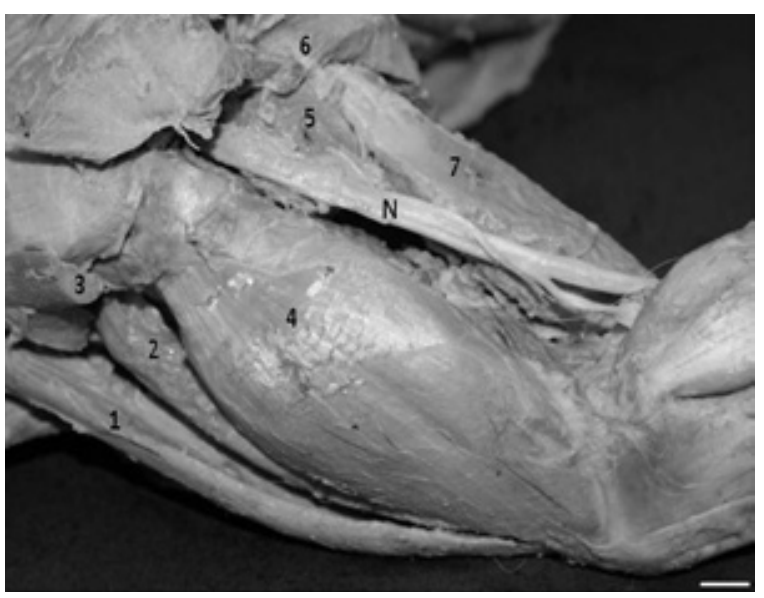

FIGURA 4. Vista lateral da coxa esquerda de $P$. cancrivoru, onde estão situados os músculos: 1) sartório; 2) retofemoral; 3) tensor da fascialata seccionado; 4) vastolateral; 5) quadrado da femoral; 6) semitendinoso; 7) semimembranoso; N) nervo isquiático. Barra $=0,5 \mathrm{~cm}$.

\section{DISCUSSÃO}

Neste trabalho, em que se estudaram os músculos da coxa de $P$. cancrivorus, os dados obtidos foram comparados com os existentes na literatura de anatomia veterinária, como cães e gatos, especificamente.
Em carnívoros domésticos, GETTY (1986) descreve os músculos do membro pélvico, enfatizando, principalmente, os cães. No entanto, cita que os músculos dos membros, especialmente os mais distais, são mais desenvolvidos no felino do que no cão, dentre outras divergências musculares, como a presença do músculo sóleo em felinos. KONIG \& LIEBICH (2002) descrevem a miologia dos carnívoros domésticos, utilizando como exemplo, em suas ilustrações, o cão, assim como SCHALLER (1999), EVANS \& DE LAHUNTA (1994), que descrevem especialmente a musculatura do cão, enfatizando sua dissecção. POSPEKO (1990) ilustra a topografia dos carnívoros domésticos. Acredita-se que as descrições musculares em carnívoros domésticos são extrapoláveis para todas as demais espécies dos Carnivora, podendo ocorrer variações relevantes ou que refletem uma conformação corporal diferente entre as espécies. É o caso das encontradas nos músculos do antebraço de mão-pelada, mais semelhantes aos músculos do antebraço do gato, por apresentar o músculo braquioradial, ausente no cão (LIMA et al., 2010). Assim como os músculos do braço e da perna de mão-pelada, que ocorrem similarmente aos músculos dos carnívoros domésticos (LIMA et al., 2009a; PEREIRA et al., 2010).

Os carnívoros domésticos apresentam o músculo quadríceps femoral consistindo em quatro porções: o músculo reto da coxa é a porção mais longa e possui inserção proximal no osso pélvico por um tendão curto, de uma tuberosidade logo cranial ao acetábulo. $\mathrm{O}$ músculo vastomedial e o músculo vastolateral possuem suas inserções proximais no lado respectivo do fêmur, próximo à extremidade proximal. Profundamente ao músculo vastolateral, com o qual ele surge, caudalmente ao músculo reto da coxa, está o músculo vastointermédio. Todas as quatro porções terminam em um tendão comum contendo a patela e inserem-se distalmente na cavidade da tuberosidade tibial (GETTY, 1986; POSPEKO, 1990; EVANS \& DE LAHUNTA, 1994; SCHALLER, 1999; KONIG \& LIEBICH, 2002), o que corresponde aos achados deste estudo, em todos os cinco espécimes de $P$. cancrivorus (Figuras 1 e 2). KONIG \& LIEBICH (2002) citam que no gato é nítida a divisão em quatro ventres musculares. Em cães essa divisão é menos evidente, assim como no mão-pelada (Figuras 1 e 2). Em Procyon cancrivorus, o músculo quadríceps da coxa é extensor da articulação do joelho. 
Autores como GETTY (1986), EVANS \& DE LAHUNTA (1994), SCHALLER (1999) e KONIG \& LIEBICH (2002) citam que em cães o músculo sartório consiste em duas porções com inserção proximal na tuberosidade da coxa. Fato corroborado pelas ilustrações destes músculos no cão, como relatado por POPESKO (1990). Em P. cancrivorus, este músculo não apresenta divisões no ventremuscular. Consiste, portanto, em uma única porção muscular, correspondendo à porção cranial do cão, similar ao gato. De acordo com GETTY (1986), este músculo não é dividido em duas porções (Figura 1). No entanto, a inserção proximal se assemelha à inserção da porção cranial do cão, na tuberosidade coxal, situando-se junto ao músculo tensor da fascialata como limite cranial da coxa, inserindo-se distalmente junto à aponeurose do músculo grácil e medialmente na margem cranial da tíbia, sendo este músculo um adutor do membro pélvico (POSPEKO, 1990; EVANS \& DE LAHUNTA, 1994; SCHALLER, 1999; KONIG \& LIEBICH, 2002). Em mão-pelada, o músculo sartório não cobre o canal femoral, sendo possível sentir-se o pulso nesse local, similarmente ao que ocorre nos carnívoros domésticos (KONIG \& LIEBICH, 2002).

Em todos os cincos espécimes utilizados no estudo, o músculo grácil apresenta inserção proximal aponeurótica no tendão sinfisial e tendão de inserção distal plano e forte, profundamente na porção caudal do músculo sartório e ao longo de todo o comprimento da margem cranial da tíbia (Figuras 1 e 2). Este atua como adutor do membro pélvico, similarmente ao cão e gato (GETTY, 1986; EVANS \& DE LAHUNTA, 1994; SCHALLER, 1999; KONIG \& LIEBICH, 2002).

KONIG \& LIEBICH (2002) afirmam que os músculos adutores se configuram diferentemente, como se verifica no cão e principalmente no gato, que apresenta somente um adutor longo. Em P. cancrivorus, verifica-se um músculo adutor curto, que se insere proximalmente no tendão sinfisial e distalmente na superfície rugosa da extremidade distal do fêmur. O músculo adutor magno insere-se proximalmente do tendão sinfisial ímpar, coberto pelo músculo grácil, relacionado ao músculo vasto medial, para inserir-se distalmente no epicôndilo medial do fêmur (Figura 1 e 2), como é citado por aqueles autores para os carnívoros. Os adutores abduzem o membro pélvico e estendem a bacia.
O músculo pectíneo no cão e gato, segundo GETTY (1986), é relativamente pequeno e fusiforme e surge do tendão pré-púbico, descendo na coxa em um sulco entre o músculo vasto medial e o músculo adutor, para formar uma inserção distal tendínea ao longo da superfície caudal do fêmur, distalmente à sua parte média. A porção medial de sua inserção une-se à porção do músculo semimembranoso. Ele aduz a coxa e gira o membro lateralmente. Estas descrições anatômicas são corroboradas por SEIFERLE (1968), EVANS \& DE LAHUNTA(1994), SCHALLER (1999) e KONIG \& LIEBICH (2002), o que da mesma forma prevalece em $P$. canvrivorus (Figuras 1 e 2 ).

GETTY (1986), EVANS \& DE LAHUNTA (1994), SCHALLER (1999) e KONIG \& LIEBICH (2002) citam que, nos carnívoros domésticos, o músculo bíceps femoral insere-se proximalmente na tuberosidade isquiático (cabeça vertebral) e no terço distal do ligamento sacrotuberal (cabeça pélvica). Insere-se distalmente por meio das fáscia lata e crural à patela, ao ligamento patelar e à borda cranial da tíbia, por meio da fáscia crural à parte subcutânea do corpo da tíbia e à tuberosidade calcânea, como verificado nesta pesquisa, em todos os espécimes de $P$. cancrivorus (Figura 3). Ele flexiona a articulação do joelho, estende o tarso e abduz o membro pélvico.

O músculo tensor da fáscia lata no gato é extenso (STANLEY et al., 2002). No cão é um músculo triangular que surge da tuberosidade da coxa para unir-se, ao nível do trocanter maior, à fáscia lata (GETTY, 1986; POSPEKO, 1990; EVANS \& DE LAHUNTA, 1994; SCHALLER, 1999; KONIG \& LIEBICH, 2002). Esta continua distal e lateralmente ao quadríceps, até a patela, o que corresponde silmilarmente aos achados desta pesquisa em P. cancrivorus (Figura 3).

O músculo semitendinoso insere-se proximalmente na tuberosidade isquiática caudal à origem do músculo bíceps da coxa, descendo caudalmente ao músculo bíceps da coxa, para ter curso medial e passar medialmente à fossa poplítea e aos músculos caudais da coxa. Insere-se distalmente por meio de um tendão forte e plano, na porção distal da borda cranial da tíbia, distalmente ao tendão do músculo grácil. Atua como flexor da articulação do joelho, extensor do quadril e da articulação tibiotársica do $P$. cancrivorus (Figuras 3 e 4), o que pode ser observado nas descrições de GETTY (1986), EVANS \& DE LAHUNTA (1994), SCHALLER (1999) e KONIG \& LIEBICH (2002). 
POSPEKO (1990) ilustra o músculo semimembranoso e KONIG \& LIEBICH (2002) descrevem-no no cão com inserção proximal na tuberosidade isquiática, caudal e medialmente ao músculo semitendinoso, tornando-se cranial ao referido músculo, ao dobrar-se medialmente para se inserir distalmente. Duas porções podem ser distinguidas, embora tenham inserções distais adjacentes. A porção cranial tende a se inserir à porção caudal, e assim se une ao epicôndilo medial do fêmur e à origem do músculo gastrocnêmio, ao passo que a porção caudal possui inserção distal no epicôndilo medial da tíbia. Estende a articulação do quadril e abduz o membro pélvico (GETTY, 1986; EVANS \& DE LAHUNTA, 1994; SCHALLER, 1999), da mesma forma como no mão-pelada.

Em todos os cinco espécimes sob exame do presente estudo, os músculos gêmeos inserem-se proximalmente à borda lateral do ísquio, permanecendo as duas partes lado a lado, apenas demarcadas pelo tendão do músculo obturador interno, com o qual elas se inserem distalmente na fossa trocantérica (Figura 4). E atuam como rotador externo da coxa, análogo às descrições de EVANS \& DE LAHUNTA (1994), SCHALLER (1999) e KONIG \& LIEBICH (2002), para os carnívoros domésticos.

O músculo quadrado femoral tem sua inserção proximal medialmente à tuberosidade isquiática, corre, cranial e distalmente, próximo ao músculo adutor, para inserir-se distalmente na fossa trocantérica, como nas descrições de GETTY (1986). Isso é corroborado por EVANS \& DE LAHUNTA (1994), SCHALLER(1999) \& KONIG \& LIEBICH (2002), para os carnívoros domésticos, o que da mesma forma foi encontrado em todos os cinco espécimes de mão-pelada. Este músculo atua como extensor da articulação coxofemoral e desloca as extremidades para trás (Figura 4).

O músculo abdutor crural caudal, presente apenas em carnívoros (KONIG \& LIEBICH, 2002), no mão-pelada apresenta inserção proximal na porção distal do ligamento sacrotuberal e distalmente, na fáscia crural, com fibras mais caudais do músculo bíceps da coxa, em conformidade com as citações de GETTY (1986), EVANS \& DE LAHUNTA (1994), SCHALLER (1999) e KONIG \& LIEBICH (2002), para os carnívoros domésticos.

No cão e gato, EVANS \& DE LAHUNTA (1994) citam que os músculos quadríceps femoral, sartório, tensor da fáscia lata são supridos por ramos do nervo isquiático e nutridos por ramos da artéria femoral. Exceto o músculo tensor da fáscia lata no tocante à inervação, intermediada pelo nervo glúteo cranial. Os músculos bíceps femoral, semitendinoso, adutores, semimembranoso, grácil, abdutor crural caudal, gêmeos, quadrado femoral, pectíneo e grácil são inervados por ramos do nervo isquiático e nutridos por ramos da artéria femoral profunda. Exceto os músculos adutores, pectíneo e grácil no tocante à vascularização, intermediada por ramos da artéria femoral, continuação caudal da artéria ilíaca interna. Os estudos destes autores não abrangem as respectivas veias que vascularizam este grupo muscular. Porém, estas citações são apresentadas em esquemas por autores como POSPEKO (1990), SCHALLER (1999) e KONIG \& LIEBICH (2002), cujos estudos abrangem toda a vascularização venosa do membro pélvico do cão e gato. Acredita-se que, no tocante à vascularização arterial e venosa dos músculos da coxa de $P$. cancrivorus, o mesmo ocorra nos respectivos músculos em mão-pelada, dada a grande similaridade morfológica destes músculos em carnívoros domésticos.

\section{CONCLUSÃO}

Com base na metodologia empregada e na análise dos resultados obtidos, constatou-se que os músculos da coxa de Procyon cancrivorus, no geral, são similares ao respectivo grupo muscular nos carnívoros domésticos, como cães e gatos, não ocorrendo divergências quanto à inserção distal e proximal dos músculos da coxa. Exceto no músculo sartório, uma vez que apresenta somente um ventre muscular e pelo fato de todos os músculos em ênfase refletirem uma conformação corporal diferente dos carnívoros domésticos. O presente estudo induz a afirmar que todos os músculos da coxa deste carnívoro silvestre obedecem ao mesmo padrão morfológico no tocante às inserções distais e proximais encontradas em carnívoros domésticos. Este estudo elucida os padrões morfológicos da miologia da coxa do Procyon cancrivorus, fato que o torna um meio que poderá contribuir em futuros estudos biomecânicos, morfofisiológicos e a outras áreas correlatas. 


\section{REFERÊNCIAS}

AVERSI-FERREIRA, T. A.; VIEIRA, L. G.; PIRES, R. M.; SILVA, Z.; PENHA-SILVA, N. Estudo anatômico dos músculos flexores superficiais do antebraço no macaco Cebus apella. Bioscience Journal, v. 22, n. 1, p. 139-144, 2006.

BARQUEZ, R. M.; DÍAZ, M. M.; OJEDA, R. A. Mamíferos de Argentina: sistemática y distribución. Tucumán: SAREM, 2006. p. 359.

CÂMARA, T.; MURTA, R. Mamíferos da Serra do Cipó. Belo Horizonte: PUC-Minas/Museu de Ciências Naturais, 2003. 60 p.

CAVAlCANTI, N. B.; RESENDE, G. M.; BRITO, L. T. L. Regeneração natural de sementes do imbuzeiro (Spondias tuberosa Arruda) no sertão de Pernambuco. Engenharia Ambiental, v. 6, n. 2, p. 342-357, 2009.

CUBAS, Z. S.; SILVA, J. C. R.; CATÃO-DIAS, J. L. CarnivoraProcyonidae (Quati, Mão-pelada, Jupará). In: Tratado de animais selvagens: medicina veterinária. São Paulo: Roca, 2006. $571 \mathrm{p}$.

DIAS, C. V. B.; SOUZA, T. D.; SILVA, S. C.; MELOTTI T.; LEITE, F. L. G. Criptorquidismo em Procyon cancrivorus (G. Cuvier, 1798) De vida livre do litoral do Espirito Santo, Brasil. In: ENCONTRO INTERNACIONAL DE MEDICINA DA CONSERVACÃO, 1., 2007. Vitória, ES. Anais... Vitória, ES, 2007.<http://institutomarcosdaniel.org.br/eimc/documentos/anais_eimc_polf $>$

DÍAZ, M. M.; BARQUEZ, R. M. Los mamíferos de jujuy. Buenos Aires, Argentina: L.O.L.A, 2002. 158 p.

DI DIO, L. J. A.; AMATUZZI, M. M.; CRICENTI, S. V. Sistema muscular. In: DI DIO, L. J. A. (Ed.). Tratado de anatomia sistêmica aplicada. São Paulo: Atheneu, 2003. p. 187-287.

EVANS, H. E.; de LAHUNTA, A. Guia para dissecção do cão. 3. ed. Rio de Janeiro: Guanabara Koogan, 1994. p. 348.

SEIFERLE, E.; BEWEGUNGSAPPARAT. In: NICKEL, R.; SCHUMMER, A.; SEIFERLE, E. Lerbuch der anatomie der haustiere. Berlim: Paul Parey, 1968. Band 1.p. 427.

FREITAS, Marco Antonio de; SILVA, Thais Figueiredo Santos. Mamíferos da Bahia: espécies continentais, Pelotas, RS: USEB, $2005.85 \mathrm{p}$.

HILDEBRAND, M.; GOSLOW, G. E.; Músculos e órgãos elétricos. In: Análise da estrutura dos vertebrados. 2. ed. São Paulo: Atheneu, 2006. 169 p.

INTERNATIONAL COMMITTEE ON VETERINARY GROSS ANATOMICAL NOMENCLATURE. Nomina anatomica veterinaria. 5. ed. Columbia, USA: Editorial Committee Hannover, 2005. $166 \mathrm{p}$.

JORGE, R. S. P.; MONICQUE, S. P.; MORATO, G. R.; SCHEFFER, K. C.; CARNIELI, P. J.; FERREIRA, F.; FURTADO, M. M.; KASHIVAKURA, C. K.; SILVEIRA L.; JACOMO, A. T. A.; LIMA, E. S.; PAULA, R. C.; MAY-JUNIOR, J. A.; KOENEMANN, J. G.; TUMELEIRO, L. K.; OLIVEIRA, É. V. Microdesgaste dentário em Procyon cancrivorus (Cuvier, 1798) (Mammalia, Procyonidae). Neotropical Biology and Conservation. v. 4, n. 2, p. 106-110, 2009.

KÖNIG, H. E.; LIEBICH, H. G. Aparelho locomotor. In: Anatomia dos animais domésticos. São Paulo: Artmed, 2002. p. 252-263.

LIMA,V. M.; PEREIRA, F. C.; . PEREIRA, K. F. Estudo morfológico dos músculos do membro torácico (braço) de mão-pelada (Procyon cancrivorus - Cuvier 1798). Veterinária Notícias, 2009a. [no prelo].

LIMA, V. M.; PEREIRA, F. C.; PEREIRA, K. F. Estudo morfológico dos músculos do antebraço de mão-pelada, Procyon cancrivorus Cuvier, 1798. Bioscience Journal, v. 26 n. 1, p. 109-114, 2010a.

LIMA, V. M.; REZENDE, A. L.; FERREIRA, J. R.; PEREIRA, K. F. Distribution of mesenteric cranial artery in the small intestine of crab-eating raccoon (Procyon cancrivorus - Cuvier 1798). Acta Scientiarum - Biological Sciences, 2009b. [no prelo].

MACHADO, A. B. M; MARTINS, C. S.; DRUMMOUNA, G. M. Lista de fauna brasileira ameaçada de extinção: incluindo as espécies quase ameaçadas e deficiente de dados. Belo Horizonte: Fundação Biodiversidade, 2005. 158 p.

MAMEDE, S. B; ALHO, J. R. Impressões do cerrado \& pantanal: subsídios para a observação de mamíferos silvestres não voadores. Campo Grande: Uniderp, 2006. p. 112-113.

MORATO, R. G.; RODRIGUES, F. H. G.; EIZIRIK, E.; MANGINI, P. R.; AZEVEDO, F. C. C. Plano de ação: pesquisa e conservação de mamíferos do Brasil. Brasília, DF: IBAMA, 2004. $52 \mathrm{p}$.

MURTA, R; CÂMARA, T. Jambreiro: fauna e flora. Belo Horizonte: R. Murta, 2006. 173 p.

ORR, R. T. Sistema muscular: biologia dos vertebrados. 5. ed. São Paulo: Roca. 1986. 186 p.

PEREIRA, F. C.; LIMA, V. M.; PEREIRA, K. F. Descrição anatômica dos músculos da perna de Procyon cancrivorus (Cuvier 1798). Biotemas, v. 23, n. 3, p. 159-165, 2010. 
POPESKO, P. Atlas de anatomia topográfica dos animais domésticos. São Paulo: Manole, 1990. v. III.

REIS, NÉLIO, R. DOS. Mamíferos do Brasil. Curitiba: Nélio R. Dos Reis, 2006. 437 p.

RODRIGUES, A. S. M.; P. AURICCHIO. Procionídeos do Brasil. São Paulo: Editora Agronômica Ceres, 1994. (Coleção Terra Brasilis, Série Zoologica - Zoo IV, Mamíferos do Brasil). 7 p.

SANTOS, A. L. Q.; MORAES, F. M.; MALTA, T. S; CARVALHO, S. F. M.; ALVES JUNIOR, J. R. F. Topografia dos colaterais do arco aórtico de um mão-pelada (Procyon cancrivorus - Gray, 1865) (Carnivora-Procyonidae). Archives of Veterinary Science, v. 9, n. 2, p. 67-72, 2004.
SCHALLER, O. Nomenclatura anatômica veterinária ilustrada. São Paulo: Manole, 1999. p. 124-129.

SISSON, S.; GROSSMAN, J. D. Músculos do carnívoro. In: GETTY, R. (Ed.). Anatomia dos animais domésticos. 5. ed. Rio de Janeiro: Guanabara Koogan, 1986. v. 2. p. 1436-1436.

STANLEY, H. DONE.; PETER, C.; GOODY, S. A.; EVANS, A.; NEIL C. STICKLAND. Anatomia veterinária do cão e do gato. São Paulo: Editora Manole, 2002. 450 p.

WIESBAUER, M. B.; GIEHL, E. L. H.; JARENKOW, J. A. Padrões morfológicos de diásporos de árvores e arvoretas zoocóricas no Parque Estadual de Itapuã, RS, Brasil. Acta Botanica Brasílica, v. 22, n. 2, p. 425-435, 2008.

Protocolado em: 7 maio 2010. Aceito em: 24 ago. 2010. 